\title{
Modelo de Identificación de Estrategias para Potencializar la Generación de Patentes a la Medida de la Institución de Educación Superior
}

\author{
Neira Yolima Figueroa Galvis ${ }^{*}$, Erika Sofía Olaya Escobar ${ }^{2}$, Hugo Fernando Castro Silva $^{3}$
}

\begin{abstract}
The transfer of knowledge and technology from universities contributes to regional development and favors innovation. The objective of this paper is to propose a model for the selection of transfer strategies adapted to the contextual characteristics of Higher Education Institutions of Colombia. For this purpose, a Qualitative Comparative Analysis (QCA) was performed, by which the combination of background conditions that generate an output is identified. The performance measure was the number of invention patents granted and the empirical application considered 184 Higher Education Institutions of Colombia. The results of the study identified ten strategic configurations that suggest different alternatives to potentiate protection through transfer mechanisms and highlights "productivity" and "innovation by territorial unit" as the best predictor of patents.
\end{abstract}

Keywords: knowledge and technology transfer; Higher Education Institutions; qualitative comparative analysis; patents.

Resumen: La transferencia de conocimiento y tecnología desde las universidades aporta al desarrollo regional y favorece la innovación. El objetivo de este trabajo es proponer un modelo para la selección de estrategias de transferencia a la medida de las características contextuales de las Instituciones de Educación Superior de Colombia. Con este propósito se realizó un análisis comparativo cualitativo (QCA), mediante el cual se identificó la combinación de condiciones antecedentes que generan un output. La medida de rendimiento fue el número de patentes de invención concedidas y la aplicación empírica consideró 184 Instituciones de Educación Superior de Colombia. Los resultados del estudio identificaron diez configuraciones estratégicas que sugieren diferentes alternativas para potencializar la protección mediante mecanismos de transferencia y destaca "la productividad" y "la innovación por unidad territorial" como mejor predictor de patentes.

Palabras clave: transferencia de conocimiento y tecnología; Instituciones de Educación Superior; análisis comparativo cualitativo; patentes.

Submitted: March $5^{\text {th }}, 2020 /$ Approved: June $15^{\text {th }}, 2020$

\section{Introducción}

La economía actual se fundamenta en el factor de producción denominado "conocimiento" y se apalanca en la innovación (Hogan, 2011). El modelo de innovación que destaca el papel de las Instituciones de Educación Superior (IES) en el proceso de transferencia es la Triple Hélice que permite entender la dinámica de interacción entre los principales actores de innovación, la Universidad, la Empresa, y el Estado (Etzkowitz \& Leydesdorff, 2000; Johnson, 2008), reconociendo a las universidades como fuente permanente de generación de conocimiento, a las empresas como fuente de soluciones a las necesidades de la sociedad, y al Estado como ente capaz de regular e incentivar mediante políticas, la transferencia entre la Universidad y la Empresa (Landry et al., 2013). Por lo anterior la relación Universidad-Empresa-Estado que se plantea en el modelo de innovación Triple Hélice, es importante para realizar la transferencia de los resultados de investigación desde las universidades a la empresa, ya que favorece el desarrollo de la tercera misión de las universidades que consiste en la difusión y divulgación de conocimiento (Etzkowitz et al., 2000; Laredo, 2007; Lockett et al., 2015; Pinheiro et al., 2015). En este sentido, las IES son líderes en el proceso de innovación y desempeñan este rol a través de la transferencia de conocimiento y tecnología (KTT).
La transferencia de conocimiento y tecnología (KTT) desde las IES, busca ofrecer soluciones a las necesidades del entorno (Etzkowitz et al., 2000; Shattock et al., 2009) y se realiza a través de la divulgación de conocimiento y tecnología hacia el sector empresarial o hacia la sociedad. Las IES con el desarrollo de las actividades de KTT, tienen la opción de comercializar en el mercado sus resultados de investigación y de esta forma acceder a nuevas fuentes de financiación (Berbegal-Mirabent \& Guerrero, 2016; Budyldina, 2018; Etzkowitz, 2003). Dentro de los resultados de investigación se destacan las patentes porque son las más apetecidas por el mercado y usualmente cuentan con un alto nivel de impacto en las mediciones y rankings sobre niveles de innovación en IES y países. A nivel general, varios autores han planteado la necesidad de realizar estudios sobre KTT para ver perspectivas alternas que permitan entender mejor su naturaleza multifacética (Bradley et al., 2013), así mismo se ha planteado la necesidad de diseñar modelos y metodologías que se adapten a la cultura y particularidades de cada región (Berbegal-Mirabent \& Guerrero, 2016; Fuquen \& Olaya-Escobar, 2018).

La generación de patentes en las IES, indica que se puede realizar de manera efectiva la transferencia de conocimiento y tecnología hacia las empresas (Han, 2017), y, teniendo en cuenta que un parámetro importante para medir el desempeño de las IES es la generación de

(1) Universidad Pedagógica y Tecnológica de Colombia, Colombia.

(2) Unidad de proyectos, Escuela Colombiana de Ingeniería Julio Garavito, (Bogotá D.C), Colombia.

(3) Escuela de ingeniería industrial, Universidad Pedagógica y Tecnológica de Colombia, Colombia.

*Autor de correspondencia: neira.figueroa@uptc.edu.co

ISSN: 0718-2724. (http://jotmi.org)

Journal of Technology Management \& Innovation (c) Universidad Alberto Hurtado, Facultad de Economía y Negocios. 
capital intelectual, las patentes tienen el máximo valor económico (Kashyap \& Agrawal, 2019). Por otro lado, las patentes concedidas a IES se consideran de mayor importancia que las concedidas a empresas, pues las primeras son más citadas y junto con las patentes gubernamentales son más generales que las asignadas a las empresas, es decir, tienen una afectación más amplia en los cambios tecnológicos posteriores de distintos grupos tecnológicos (Schmid \& Fajebe, 2019).

La transferencia favorece principalmente a los países que logran desarrollar estrategias adecuadas para llevar el conocimiento y la tecnología al mercado y a la sociedad, pues de esta manera se potencializa el desarrollo económico, social, industrial y tecnológico de las regiones y países (Etzkowitz et al., 2000; Shattock et al., 2009), pero principalmente del entorno más cercano a las IES (Carree et al., 2015; Hamdoun et al., 2018). La KTT desde las IES no es tarea fácil, pues desde su dinámica de generación de conocimiento y tipologías de resultados de investigación, deben seleccionar las estrategias apropiadas de transferencia de tal modo que respondan a su rol misional y a la vez generen beneficios económicos y sociales a sus comunidades (Chang et al., 2016; Linton, 2018; Philpott et al., 2011; Toscano et al., 2017).

La estrategia de KTT es un tema relativamente nuevo en la literatura empresarial, es un ingrediente primordial en la innovación y un salto hacia un futuro desconocido; se considera como el enfoque general que una organización acoge para alinear los recursos propios y las capacidades de conocimiento con los requisitos de carácter intelectual que conforman su estrategia de negocios (Bolisani \& Bratianu, 2017; Zack, 1999). La estrategia de KTT aplica los procesos de conocimiento a un dominio de conocimiento para lograr un objetivo estratégico que no puede separarse de la estrategia general de la empresa (Eisenhardt \& Santos, 2012; Von Krogh et al., 2001). Así mismo, es un conjunto de opciones y pautas generales que tienen en cuenta la base de conocimiento organizacional junto con la capacidad de manipular los recursos propios cognitivos para hacer el mejor uso de los activos de conocimiento y lograr una ventaja competitiva (Bierly \& Chakrabarti, 1996; Bolisani \& Bratianu, 2017; Kasten, 2007).

Para generar estrategias de transferencia, los estrategas pueden emplear capacidades estratégicas de acuerdo a las circunstancias del entorno y aplicar un "procedimiento formal para lograr un resultado articulado en forma de un sistema integrado de decisiones"(Mintzberg, 1993), que puede basarse en el pensamiento racional, lineal y determinista para descomponer el objetivo en componentes o mecanismos y asignar recursos que permitan el logro de cada uno (Bolisani \& Bratianu, 2017; Brătianu, 2015).

A nivel global, las IES protegen cada vez más sus invenciones, y el fortalecimiento de esta protección intelectual, está afectado por la aprobación e implementación de leyes que mejoren la transferencia de tecnología y faciliten la expansión de las patentes académicas, un ejemplo de esta normatividad es la ley Bayh-Dole promulgada en Estados Unidos en los años ochenta que permitió la apropiación privada de invenciones generadas por las IES de ese país. En muchos países de la OCDE, se han realizado reformas regulatorias relacionadas con la financiación de la investigación para permitirle a las IES que posean y otorguen licencias a la propiedad intelectual que se genera con fondos del gobierno. En Austria, Dinamarca, Alemania y Japón se ha abolido el privilegio del profesor de poseer patentes y este derecho es ahora de las IES, quienes proporcionan apoyo a los profesores para patentar y otorgan una parte de regalías a cambio. Sin embargo, las regulaciones no son suficientes y para mejorar las actividades de patentamiento se destaca la importancia de los incentivos tanto para instituciones como para investigadores y la presencia de masa crítica en la gestión de la propiedad intelectual (WIPO, 2020). Aunque a nivel mundial se han diseñado e implementado distintas normas similares, los índices de transferencia universitaria no se han incrementado de forma significativa en todas las universidades, y predomina en algunas, como en el caso de Latinoamérica un modelo transferencia escaso, donde muchas veces las invenciones se quedan sin ser transferidas (Fuquen \& Olaya-Escobar, 2018).

A nivel de Latinoamérica, la Comisión Económica para América Latina y el Caribe (CEPAL) indicó que existe una inversión en investigación y desarrollo (I+D) ínfima, que la región realiza el 2.8\% del gasto mundial en $\mathrm{I}+\mathrm{D}$, que el nivel de patentamiento de la región es bajo y los no residentes solicitan más patentes que los residentes, mientras en las economías avanzadas sucede lo contrario y que sólo el 2\% de las mejores universidades del mundo son latinoamericanas (CEPAL, 2016). Respecto al tema de patentamiento, la Organización Mundial de Propiedad Intelectual (OMPI) indicó que para el año 2018 recibió a nivel mundial 3.3 millones de solicitudes de patentes, que revelan un incremento de 5.2\% respecto a 2017, y de este valor total, el 66.8\% corresponde a Asia, seguido por Norte América (19\%), Europa (10.9\%), América Latina y el Caribe (1.7\%), Oceanía (1.1\%) y África (0.5\%), donde las tres últimas regiones tan sólo ocupan un 3.3\% del total de solicitudes (OMPI, 2019). Por otra parte se encontró que las universidades más innovadoras del mundo que cuentan con un flujo constante de patentes están ubicadas en América del Norte con 48 universidades, seguido por Europa (27), Asia (23), Oriente Medio (2) y América Latina África y Oceanía (0) (Reuters, 2018). Lo anterior refleja que en América Latina y el Caribe los esfuerzos en innovación son bajos y la participación en el mercado de conocimiento tanto de la región como de las universidades es marginal y se aleja del mundo desarrollado.

En el contexto anterior las IES latinoamericanas tienen muchos retos y desafíos por superar, la Conferencia Regional de Educación Superior (CRES), en junio de 2018, indicó que América Latina integra la cadena de los eslabones de bajo valor agregado y en la transición a economías ambientalmente sostenibles es un factor fundamental el enfoque al aprendizaje y la innovación para la transformación tecnológica y el potenciamiento de aspectos como el de la industria 4.0. Igualmente indicó que con el auge de los procesos de innovación las universidades tienen un papel destacado como productoras de conocimiento valioso para el desarrollo de la economía, dado que el conocimiento científico y tecnológico está más demandado que en otras épocas. Adicionalmente indicó que las universidades latinoamericanas como el principal agente ejecutor de I+D, cuentan con el 75\% de los investigadores radicados en las universidades y respecto a esta cifra se puede aprovechar este indicador para potencializar la I+D e innovación y evitar la fuga de cerebros regionales a países donde si se den suficientes espacios y garantías para la investigación y transferencia (CRES, 2018a, 2018b). 
En la actualidad no existe un modelo de selección de estrategias de transferencia claro y definido que se ajuste a la medida de las condiciones de IES de países con bajo volumen de producción académica, bajo desarrollo tecnológico, y bajos recursos de financiación como es el caso de la mayoría de los países con nivel de ingreso medio-alto ${ }^{1}$ (a excepción de China) donde se ubica Colombia. Por otra parte, las IES de estos países muchas veces generan las soluciones idóneas y las tecnologías que su industria necesita, pero los resultados de investigación se quedan en las IES y no son transferidos a la sociedad (Fuquen \& Olaya-Escobar, 2018).

Este trabajo aborda la KTT de las IES en Colombia desde la perspectiva de las ciencias económicas y administrativas. El interés en el tema viene dado por los bajos niveles de transferencia que se traducen en bajos niveles de innovación y desarrollo y, por tanto, en nuevos desafíos para la educación y las IES del país. Por lo anterior, el propósito de este trabajo es proponer un modelo para la identificación de estrategias que potencialicen la generación de patentes como mecanismo de protección para la posterior transferencia en las IES de Colombia. La pregunta que se busca responder es la siguiente: ¿Cómo podrían las Instituciones de Educación Superior generar patentes teniendo en cuenta diferentes condiciones antecedentes?

Este documento se encuentra organizado de la siguiente manera: primero la presentación de los factores y outputs de transferencia encontrados en la literatura. Segundo, la metodología fundamentada en el análisis QCA. Tercero, los resultados del trabajo. Cuarto, la discusión y quinto las conclusiones.

\section{Factores y Outputs de transferencia}

A partir de un análisis de la literatura especializada en el tema se identificaron 7 factores que inciden en la KTT: 1) ambientales, que se refieren a las características socioeconómicas y de innovación de la región; 2) legales, asociados a las restricciones y normas; 3) capital humano, relacionado con los recursos humanos y aptitudes del personal; 4) estructurales, definido como las características propias del funcionamiento a nivel interno de las IES y de las Oficinas de Transferencia; 5) financieros, como las fuentes de financiación de actividades de Investigación y Desarrollo (I+D); 6) relacionales, como las redes de contacto entre los actores; y 7) de productividad, como los resultados de I+D (Berbegal-Mirabent \& Solé-Parellada, 2011; Olaya-Escobar et al., 2014). Así mismo, se identificaron 5 outputs de KTT: patentes, licencias o acuerdos de regalías, investigación en colaboración, investigación por contrato, y spin-off (Arenas \& González, 2018; Berbegal-Mirabent \& Solé-Parellada, 2011; Franco \& Haase, 2015; Olaya-Escobar et al., 2014); dentro de los cuales se destaca la patente como el mecanismo elegido por excelencia para la protección de resultados de investigación (Berbegal-Mirabent \& Solé-Parellada, 2011). A continuación, en la Tabla 1 se presentan los outputs de KTT junto con los principales indicadores identificados.

Tabla 1. Indicadores por output de transferencia. Fuente: (Figueroa-Galvis et al., 2019a)

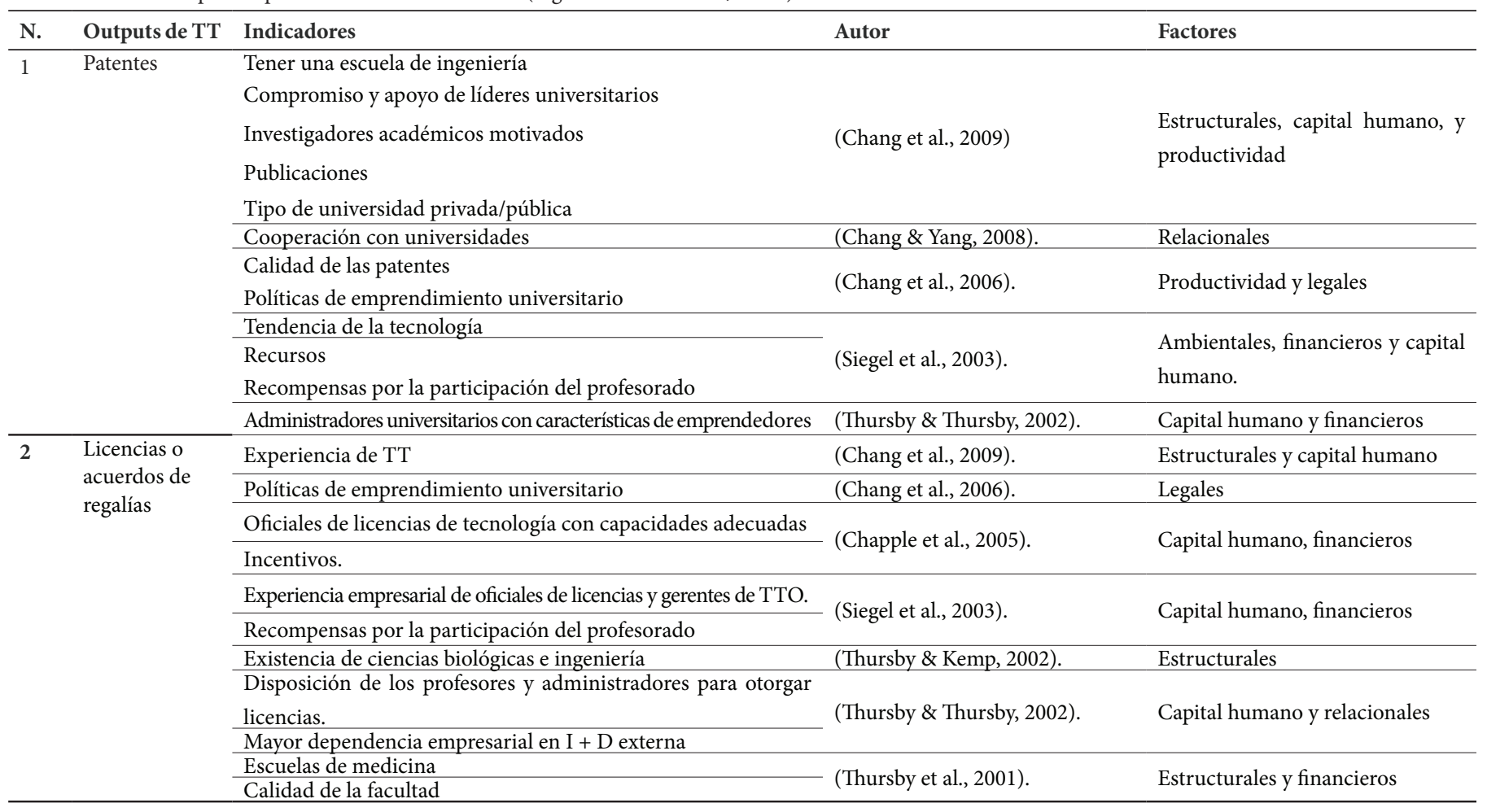

${ }^{1}$ Según el Banco Mundial, los países con nivel de ingreso medio-alto son aquellos que generan un Ingreso Nacional Bruto (INB) per cápita entre US\$3.956 y US12.235. En el año 2008 Colombia ingresó al grupo de clasificación medio-alto y ha permanecido ininterrumpidamente en esta clasificación hasta el informe del año 2018 (The World Bank, 2018 ). La clasificación de países por nivel de ingreso es utilizada por la OMPI en la presentación anual de los índices globales de innovación (GII) (Cornell University et al., 2017). 


\begin{tabular}{lllll}
\hline $\mathbf{N}$. & Outputs de TT & Indicadores & Autor & Factores \\
\hline $\mathbf{3}$ & $\begin{array}{l}\text { Investigación } \\
\text { en colabora- } \\
\text { ción }\end{array}$ & Existencia de otras universidades en la región & (Wright et al., 2008). & Ambientales y capital humano \\
\cline { 2 - 5 } & Masa crítica en el equipo investigador & Investigaciones cooperativas especialmente con empresas privadas & (HAN \& KIM, 2016). & Relacionales \\
\hline $\mathbf{4}$ & $\begin{array}{l}\text { Investigación } \\
\text { por contrato }\end{array}$ & Receptividad a los contratos industriales & (Thursby \& Thursby, 2002). & Capital humano \\
\hline
\end{tabular}

$5 \quad$ Spin off - Start Presencia de incubadoras

ups $\quad$ Fondo empresarial de la universidad para fomentar la partici-

pación en Spin-off

Proactividad

Toma de riesgos

Rasgos empresariales reconocidos

(Chang et al., 2009).

Estructurales, financieros, capital

Apoyos organizacionales

Capacidades empresariales personales

Disponibilidad de tiempo

Tipo de universidad privada/pública.

Políticas de emprendimiento universitario

Colaboraciones IES-industria

(Chang et al., 2006).

Legales y relacionales

Capacidades de los funcionarios en empresas spinning-out.

Recursos para el gasto en protección de propiedad intelectual

Capacidades de desarrollo de negocios

Experiencia y habilidades del personal de TT

Personal adecuadamente capacitado

Pagos de regalías.

(Lockett \& Wright, 2005)given

concerns regarding licensing and

the university's desire to maximize the returns to intellectual property (IP.

(Markman et al., 2005)entrepreneurship theory and research on the role of the UTTO in business incubation and new venture for-

TTO con fines de lucro. mation is sparse. To move the research along, we use grounded theory to build a framework to address two questions: (a.

\begin{tabular}{|c|c|c|}
\hline Calidad de los profesores & \multirow{8}{*}{ (O’Shea et al., 2005). } & \multirow{8}{*}{$\begin{array}{l}\text { Capital humano, financieros, es- } \\
\text { tructurales, ambientales, relacio- } \\
\text { nales }\end{array}$} \\
\hline Tamaño y orientación de fondos de ciencia e ingeniería & & \\
\hline Capacidad comercial & & \\
\hline Características organizativas de las universidades & & \\
\hline Cultura de apoyo comercial. & & \\
\hline Asociación de apoyo financiero con la industria y el gobierno. & & \\
\hline Desarrollo de la ciencia y la ingeniería. & & \\
\hline Desarrollo de infraestructura comercial. & & \\
\hline Eminencia intelectual. & \multirow{3}{*}{ (Di Gregorio \& Shane, 2003). } & \multirow{3}{*}{$\begin{array}{l}\text { Capital humano, legales, estruc- } \\
\text { turales. }\end{array}$} \\
\hline Políticas de inversiones en empresas de nueva creación. & & \\
\hline Baja participación de inventores en regalías. & & \\
\hline
\end{tabular}

Complementario a lo anterior, se encontró que los factores de mayor incidencia para potencializar la KTT en el contexto de las IES de Colombia son cuatro: Estructural, Productividad, Relacional y Capital humano e investigación (Figueroa-Galvis et al., 2019a, 2019b). Los cuales fueron identificados a través de un análisis factorial confirmatorio (AFC) que se apoyó en un análisis factorial exploratorio (AFE) y un análisis de información de la literatura internacional, de bases de datos de Colombia y de algunos rankings internacionales sobre Ciencia, Tecnología e Innovación. Adicionalmente, con las medidas de validez discriminante del AFC y los índices de bondad de ajuste obtenidos en el estudio en mención, se confirmó que se pueden construir modelos tomando como referencia estos factores identificados.

\section{Metodología}

El análisis comparativo cualitativo (QCA) es un método que permite realizar el análisis formal y sistemático de la causalidad, así mismo provee herramientas para mejorar el análisis empírico cuando se realizan comparaciones de pequeños números de casos. Este método ayuda al investigador a representar y sintetizar los datos con los que cuenta para mejorar el diálogo entre las ideas y la evidencia empírica (Gandini, 2014). Una de las ventajas es que a diferencia de otros métodos cuantitativos utilizados en las ciencias sociales para el análisis de datos (ANOVA, ARM, SEM), que por tradición estudian las variables de forma independiente, el QCA permite estudiar los casos 
bajo la idea que las relaciones causales se entienden mejor entre conjuntos teóricos que entre correlaciones, de tal forma que busca a partir de configuraciones de condiciones causales la obtención de una salida o resultado (Fernández Guerrero et al., 2018; Fiss, 2011; Ragin \& Fiss, 2008). Por lo general para el análisis QCA se requieren de diez a cincuenta muestras, lo cual se convierte en otra ventaja porque permite ofrecer respuestas validas a muestras pequeñas, sin embargo, un buen número de estudios usa QCA en muestras grandes y afirma que el análisis es igual de concluyente (Fiss, 2011; Woodside, 2012). Una de las desventajas de utilizar este método es que, debido a que es una herramienta metodológica relativamente nueva, en la literatura aún falta el establecimiento de reglas de buenas prácticas. Sin embargo, se han tomado las orientaciones sobre buenas prácticas que plantea Schneider \& Wagemann (Schneider \& Wagemann, 2010) en el desarrollo metodológico de este estudio.

El QCA hace uso del algebra booleana para observar la relación que existe entre todas las combinaciones de variables binarias predictoras y una variable de salida, y genera como resultado un número de configuraciones que sugieren distintos caminos teóricos para lograr la salida (Longest \& Vaisey, 2008). El proceso de QCA consta básicamente de los siguientes pasos, primero la calibración de las variables, que consiste en adecuar las variables al lenguaje binario, donde el rango de miembro del set es 1 , de no miembro del set 0.0 y de ambigüedad de 0.5 (Ragin, 2008). De acuerdo al tipo de variable se realiza la calibración, en el caso de variables dicotómicas la transformación es en términos Crisp, donde 1 = inclusión y 0 = no-inclusión, en el caso de variables continuas la calibración se realiza en términos de conjuntos difusos Fuzzy, donde los datos originales se analizan con puntos de corte que típicamente son de 0.95 como miembro del set, 0.05 no miembro del set y 0.5 ambigüedad (Ragin, 2006). El segundo paso consiste en realizar el análisis de condiciones necesarias, para asegurarse que ninguna de las variables predictoras o antecedentes es condición necesaria para lograr la salida (Schneider \& Wagemann, 2010), el tercer paso consiste en la elaboración de la tabla de verdad, donde se generan todas las combinaciones lógicas posibles con las condiciones definidas previamente (Fiss, 2011) y finalmente el software calcula las configuraciones mínimas que generan la salida haciendo uso del algoritmo Quine-McCluskey, dichas configuraciones tienen en cuenta dos parámetros principales que son cobertura, que indica la relevancia de la solución y consistencia que indica el grado en que para generar la salida los casos comparten condiciones similares (Ragin, 2008).

Dentro de las herramientas de software desarrolladas para QCA, se encuentran: QCA-DOS para set de datos Crisp (nítidos), FSQCA para set de datos Fuzzy (difusos), FSQCA 2.0 para set de datos Crisp y Fuzzy, TOSMANA para set de datos Crisp y multivalores, entre otros. Para este estudio se seleccionó FSQCA 2.0 (Ragin et al., 2006), porque integra los dos sets de datos tanto Fuzzy como Crisp que corresponden al set de datos a utilizar en este análisis. QCA es adecuado para este estudio, debido a que el interés de este trabajo está orientado a identificar las combinaciones de factores o variables necesarias para lograr patentes de invención concedidas, más no es tan relevante identificar los factores necesarios, dado que ya se identificaron previamente y para ese caso si se podrían utilizar otra variedad de métodos.

\section{Muestra}

La muestra corresponde a 184 IES, se llegó a este tamaño mediante la depuración de la base de datos reportadas en el Sistema Nacional de Educación Superior (SNIES) de Colombia, año 2017. Se eliminó duplicidad de información hasta 376 IES, luego se seleccionaron 360 IES activas y según clasificación por tipo de institución se descartaron las categorizadas en Otras. Las principales características de las IES seleccionadas se presentan en la tabla 2. Respecto al número de patentes concedidas, se tomó la información de la Superintendencia de Industria y Comercio de Colombia (SIC) sobre el ranking de patentes de las universidades colombianas de los años 2000 a 2017.

Tabla 2. Principales características de las IES seleccionadas. Fuente: Elaboración propia.

\begin{tabular}{|c|c|c|c|}
\hline & Carácter académico & Número & $\%$ \\
\hline & $\begin{array}{l}\text { Universidad } \\
\end{array}$ & 84 & 45,65 \\
\hline \multirow[t]{2}{*}{ Sector } & & Número & $\%$ \\
\hline & Oficial & 57 & 30,98 \\
\hline \multirow[t]{7}{*}{ Tipo de Institución } & & Número & $\%$ \\
\hline & Doctoral & 14 & 7,61 \\
\hline & Instituciones con 2 a 4 áreas de conocimiento en pregrado & 58 & 31,52 \\
\hline & Instituciones con 5 a 8 áreas de conocimiento en pregrado & 24 & 13,04 \\
\hline & Maestría & 32 & 17,39 \\
\hline & Universidades con 2 a 4 áreas de conocimiento en pregrado & 11 & 5,98 \\
\hline & Universidades con 5 a 8 áreas de conocimiento en pregrado & 26 & 14,13 \\
\hline \multirow{2}{*}{ Acreditación de alta calidad } & & Número & \\
\hline & Con Acreditación de Alta Calidad & 51 & 27,71 \\
\hline Número de patentes concedidas & IES sin patentes & 149 & $81 \%$ \\
\hline
\end{tabular}




\section{Outcome y variables antecedentes}

- Outcome: como outcome o resultado a explicar en el modelo, se seleccionó el número de patentes de invención concedidas a las IES colombianas. El outcome se seleccionó teniendo en cuenta que normalmente es un producto fácilmente cuantificable y el mecanismo de protección de resultados de investigación por excelencia (Berbegal-Mirabent \& Solé-Parellada, 2011), así mismo, que tiene un alto impacto en la medición de innovación de las IES (Reuters, 2018), que involucra como entrada otros tipos de resultados de investigación, que existe facilidad de acceso a su información estadística, que es una apuesta del país fortalecer el patentamiento y que es un paso previo para el licenciamiento y la conformación de empresas de base tecnológica.

- Variables antecedentes: se tomaron siete variables antecedentes, cuatro corresponden a factores o variables latentes que inciden en la KTT y tres a variables que se considera afectan el contexto de las IES en Colombia (Ver tabla 3 y figura 1). A continuación, se describe el proceso de obtención:

Factores o variables latentes que inciden en la KTT: se tomaron cuatro factores extraídos con un análisis factorial confirmatorio
(CFA) realizado en un estudio previo (Figueroa-Galvis et al., 2019a, 2019b). Se logró la obtención de estos factores tomando inicialmente como referencia la identificación de factores que inciden en la KTT según la literatura internacional (Ver primer apartado de este documento, factores y outputs de transferencia), luego se tomaron bases de datos con estadísticas y rankings sobre actividades de KTT en las IES de Colombia y de acuerdo a esta información se elaboró un primer análisis factorial exploratorio que luego fue corroborado con un CFA. Los nombres de cada factor están asociados al contenido de los mismos y se relacionan con el referente existente en la literatura. Debido a que Colombia no cuenta con información robusta de estadísticas que respondan a todos los factores que se encuentran en la literatura y que afectan la KTT, sólo se logró traer al contexto cuatro factores: estructural, productividad, relacional y capital humano e investigación (Figueroa-Galvis et al., 2019a, 2019b), ver descripción en la tabla 3.

Variables que afectan el contexto de las IES: se consideró importante complementar la información de los cuatro factores antecedentes y fortalecer el enfoque contextual de este estudio con las siguientes variables: unidad territorial de domicilio, acreditación de alta calidad, innovación por unidad territorial (Ver descripción en la tabla 3).

Tabla 3. outcome y variables antecedentes del modelo.

\begin{tabular}{|c|c|c|c|c|}
\hline & ITEM & DEFINICIÓN DE ITEM & $\begin{array}{l}\text { DESCRIPCIÓN DE CADA ITEM DENTRO } \\
\text { DEL ESTUDIO }\end{array}$ & $\begin{array}{l}\text { CODIFICACIÓN } \\
\text { ASIGNADA A CADA ITEM }\end{array}$ \\
\hline OUTCOME & Patentes de invención & Output de KTT seleccionado. & Corresponde a patentes de invención concedidas. & Patentesc \\
\hline \multirow[t]{7}{*}{$\begin{array}{l}\text { VARIABLES } \\
\text { A N T E C E - } \\
\text { DENTES }\end{array}$} & Estructural & $\begin{array}{l}\text { Características propias del funcio- } \\
\text { namiento a nivel interno de las IES. } \\
\text { (Berbegal-Mirabent \& Solé-Parellada, } \\
\text { 2011) (Olaya-Escobar et al., 2014) }\end{array}$ & $\begin{array}{l}\text { Corresponde al factor o variable latente que } \\
\text { abarca la información sobre: la posición de las } \\
\text { IES en rankings internacionales, años de crea- } \\
\text { ción y vigencia de la acreditación. }\end{array}$ & Estructural \\
\hline & Productividad & $\begin{array}{l}\text { Resultados de I+D que condicionan } \\
\text { a otros. (Berbegal-Mirabent \& Solé- } \\
\text { Parellada, 2011) (Olaya-Escobar et } \\
\text { al., 2014) }\end{array}$ & $\begin{array}{l}\text { Corresponde al factor o variable latente que } \\
\text { abarca la información sobre: modelos de utili- } \\
\text { dad concedidos y presentados, así como paten- } \\
\text { tes presentadas y publicadas. }\end{array}$ & Productividad \\
\hline & Relacional & $\begin{array}{l}\text { Redes de contacto entre los actores } \\
\text { (Olaya-Escobar et al., 2014) }\end{array}$ & $\begin{array}{l}\text { Corresponde al factor o variable latente que } \\
\text { abarca la información sobre: coautorías y ci- } \\
\text { taciones por artículo publicado en la base de } \\
\text { datos académica Web of Science (WOS) y coau- } \\
\text { torías y citaciones por artículo publicado en la } \\
\text { base de datos SCOPUS. }\end{array}$ & Relacional \\
\hline & $\begin{array}{l}\text { Capital humano e inves- } \\
\text { tigación }\end{array}$ & $\begin{array}{l}\text { Relacionado con los recursos huma- } \\
\text { nos y aptitudes del personal. (Ber- } \\
\text { begal-Mirabent \& Solé-Parellada, } \\
\text { 2011) (Olaya-Escobar et al., 2014) }\end{array}$ & $\begin{array}{l}\text { Corresponde al factor o variable latente que } \\
\text { abarca la información sobre: apropiación social } \\
\text { de conocimiento docente, formación del recur- } \\
\text { so humano docente y generación de nuevo co- } \\
\text { nocimiento docente. }\end{array}$ & Capitalhi \\
\hline & $\begin{array}{l}\text { Unidad territorial de } \\
\text { domicilio }\end{array}$ & $\begin{array}{l}\text { La presencia de las IES en cada una } \\
\text { de las divisiones político-adminis- } \\
\text { trativas de Colombia. }\end{array}$ & $\begin{array}{l}\text { Corresponde a la diferencia entre las IES ubica- } \\
\text { das en una unidad territorial distinta al distrito } \\
\text { capital de Bogotá, y a las ubicadas en Bogotá*. }\end{array}$ & Domicilio \\
\hline & $\begin{array}{l}\text { Acreditación de alta ca- } \\
\text { lidad }\end{array}$ & $\begin{array}{l}\text { La certificación de acreditación de } \\
\text { alta calidad de las IES de Colombia. }\end{array}$ & $\begin{array}{l}\text { Corresponde a la diferencia entre IES colom- } \\
\text { bianas que cuentan con acreditación y sin acre- } \\
\text { ditación de alta calidad. }\end{array}$ & Acreditación \\
\hline & $\begin{array}{l}\text { Innovación por unidad } \\
\text { territorial }\end{array}$ & $\begin{array}{l}\text { La innovación por cada una de las } \\
\text { divisiones político-administrativas } \\
\text { de Colombia. }\end{array}$ & $\begin{array}{l}\text { Medida que indica la posición de innovación de } \\
\text { cada unidad territorial de Colombia* }\end{array}$ & Innovación \\
\hline
\end{tabular}


Figura1. Representación relacional de las variables antecedentes con el outcome

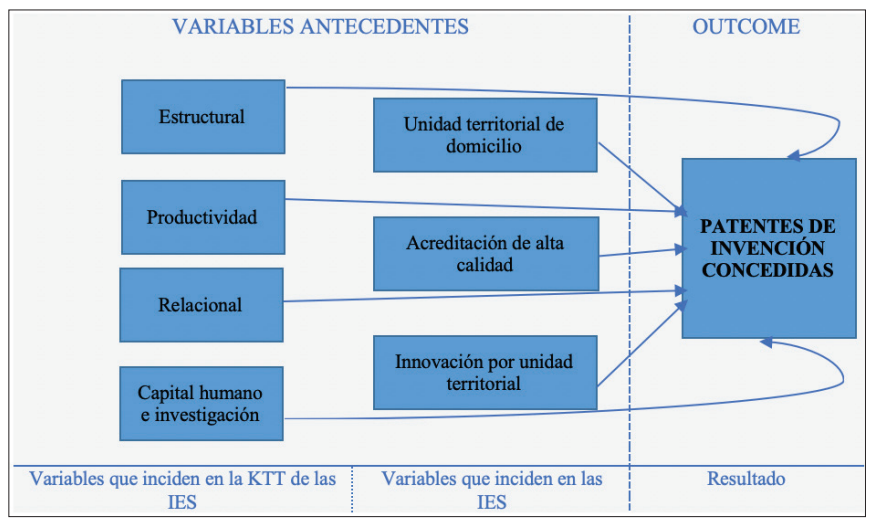

\section{Calibración de datos}

Teniendo en cuenta las siete condiciones antecedentes definidas y de acuerdo a las recomendaciones de calibración de Ragin (Ragin, 2006) se identificaron las variables "Domicilio" y "Acreditación" como Crisp, es decir variables dicotómicas donde 0 indica que están fuera del set de datos y 1 dentro del set. Respecto a los cuatro factores que corresponden a "Estructural", "Productividad", "Relacional" y "Capitalhi" junto con la variable "Innovación" se realizó la identificación Fuzzy porque son variables continuas asociadas a datos difusos que generalmente se calibran entre 0.05 y 0.95 . En la Tabla 4 se detallan los puntos de corte utilizados para cada tipo de variable.

Figura1. Elaboración propia.

Tabla 4. Definición de variables y valores de calibración. Fuente: Elaboración propia.

\begin{tabular}{|c|c|c|c|c|c|}
\hline \multirow[b]{2}{*}{ Variable } & \multirow{2}{*}{$\begin{array}{c}\text { Código de la } \\
\text { variable }\end{array}$} & \multirow[b]{2}{*}{ Descripción } & \multicolumn{3}{|c|}{ Valores del umbral de membresía } \\
\hline & & & $\begin{array}{c}\text { Fuera de set } \\
(0.05)\end{array}$ & $\begin{array}{c}\text { Punto de indefinición } \\
(0.5)\end{array}$ & $\begin{array}{c}\text { Dentro del set } \\
(0.95) \\
\end{array}$ \\
\hline Resultado & Patentesc & Patentes de invención & 0 & 0 & 6 \\
\hline \multirow{4}{*}{ Antecedentes } & Productividad & Factor productividad & $-0,754260004$ & $-0,176950235$ & 1,466521756 \\
\hline & Relacional & Factor relacional & $-0,926832207$ & $-0,187927076$ & 1,674539717 \\
\hline & Acreditación & Acreditada de alta calidad $(0=$ no, $1=$ sí $)$ & 0 & & 1 \\
\hline & Innovación & Innovación por unidad territorial de domicilio & 1 & 2 & 19.85 \\
\hline
\end{tabular}

\section{Resultados}

Se realizó el análisis de condiciones antecedentes sobre el outcome, es decir el efecto que cada variable antecedente tiene sobre la variable "Patentesc". Se revisó que el valor de la consistencia por cada variable no superara el umbral de 0.9 (Schneider \& Wagemann, 2010), y de esta manera se verifico que las variables no son condición necesaria para obtener el resultado. En la Tabla 5, se presentan los valores de consistencia y cobertura por cada variable.

Tabla 5. Análisis de condiciones necesarias. Fuente: Elaboración con fs/QCA.

\begin{tabular}{lll}
\hline condiciones probadas & consistencia & cobertura \\
\hline Outcome: Patentesc & & 0.843605 \\
\hline Estructural & 0.743334 & 0.758477 \\
$\sim$ Estructural & 0.694697 & 0.925625 \\
Productividad & 0.756031 & 0.814686 \\
$\sim$ Productividad & 0.798613 & 0.773573 \\
Relacional & 0.612950 & 0.683776 \\
$\sim$ Relacional & 0.686981 & 0.820350 \\
Capitalhi & 0.705538 & 0.759120 \\
$\sim$ Capitalhi & 0.711300 & 0.543143 \\
Domicilio & 0.371325 & 0.564649 \\
$\sim$ Domicilio & 0.628675 & 0.682745 \\
Acreditación & 0.340072 & 0.508045 \\
$\sim$ Acreditación & 0.659928 & 0.766448 \\
Innovación & 0.626917 & 0.716409 \\
$\sim$ Innovación & 0.701436 & \\
\hline
\end{tabular}

*El símbolo indica la ausencia de la condición. 
A continuación, en la Tabla 6 se presenta la solución intermedia recomendada por Ragin (Ragin, 2008), para este caso la solución es de diez configuraciones que al presentarse pueden generar la salida "patentes de invención concedidas". En la solución se utilizaron siete condiciones antecedentes o variables, y en la tabla de verdad un límite de frecuencia de 4 con la configuración de consistencia predeterminada de 0.8 . El número de configuraciones obtenidas da a entender que no existe una única solución para explicar el outcome. Se graficó la solución de acuerdo a las indicaciones de Ragin y Fiss (Ragin \& Fiss, 2008), donde el circulo negro indica la presencia de la condición, el circulo blanco la ausencia y la celda sin registro la ambigüedad de la condición (ver Tabla 6). El resultado arroja un dato de cobertura y consistencia adecuado, la cobertura general de la solución es de 0.672527 con una consistencia general de 0.959855 , que supera el umbral de 0.4 y 0.8 respectivamente, y que indica que las diez configuraciones explican el $67 \%$ de la muestra con una consistencia de $96 \%$, siendo por lo anterior el modelo válido.

Tabla 6. Configuraciones de condiciones antecedentes suficientes de entrada. Fuente: Elaboración con fs/QCA.

\section{Condiciones Antecedentes Cobertura}

\begin{tabular}{|c|c|c|c|c|c|c|c|c|c|c|}
\hline Configuración $\mathbf{N}^{\circ}$ & 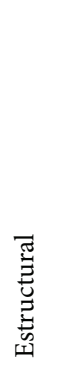 & 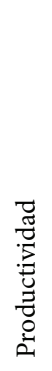 & 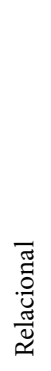 & 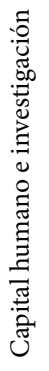 & 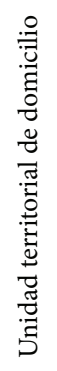 & 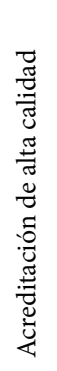 & 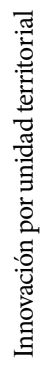 & Fila & Único & Consistencia \\
\hline 1 & $\circ$ & $\bullet$ & $\circ$ & ० & & $\circ$ & $\bullet$ & 0.284305 & 0.065631 & 0.972278 \\
\hline 2 & $\bullet$ & $\circ$ & $\bullet$ & $\bullet$ & & $\bullet$ & $\bullet$ & 0.117883 & 0.026467 & 0.948154 \\
\hline 3 & $\bullet$ & & $\bullet$ & $\bullet$ & ○ & $\bullet$ & $\bullet$ & 0.114953 & 0.038969 & 0.980017 \\
\hline 7 & $\circ$ & $\bullet$ & $\bullet$ & ○ & $\circ$ & $\circ$ & $\circ$ & 0.151284 & 0.030765 & 0.988513 \\
\hline 8 & $\bullet$ & $\circ$ & $\circ$ & $\circ$ & $\circ$ & $\circ$ & $\bullet$ & 0.166129 & 0.011134 & 0.975344 \\
\hline 9 & $\bullet$ & $\bullet$ & ○ & ○ & $\bullet$ & ○ & ○ & 0.102158 & 0.043364 & 0.995243 \\
\hline 10 & $\bullet$ & $\bullet$ & $\bullet$ & $\bullet$ & $\bullet$ & $\bullet$ & 0 & 0.039652 & 0.024221 & 0.987835 \\
\hline
\end{tabular}

Cobertura de la solución: 0.672527

Consistencia de la solución: 0.959855

El modelo planteado en QCA, inició con la identificación en la literatura de factores claves de transferencia que luego fueron traídos y validados estadísticamente en el contexto colombiano, para finalmente convertirse en la información de entrada de un modelo de análisis comparativo cualitativo (Estructural, Productividad, Relacional, Capital Humano e investigación, unidad territorial de domicilio, Acreditación de alta calidad e Innovación por unidad territorial). El modelo contiene diez configuraciones que se traducen en diez mecanismos estratégicos de KTT compuestos por la combinación de una serie de condiciones antecedentes o de entrada que permiten de acuerdo a las características de las IES colombianas obtener patentes, es decir el output con mayor impacto en las mediciones de innovación a nivel mundial y que se encuentra presente en las IES colombianas. Por lo anterior las IES pueden alinear sus recursos y capacidades de conocimiento con su estrategia institucional que por lo general se apoya en la misión, visión, políticas y prioridades estratégicas para seguir el camino que mejor se adapte a su realidad y generar ventaja competitiva en la economía actual del conocimiento.

Al observar las dos configuraciones que presentan mayor cobertura de fila según recomendación de Ragin (Ragin, 2008), se encuentra que las configuraciones uno (1) y seis (6) con cobertura por fila de $28 \%$ y $16.7 \%$ son las que aportan mayor significancia al resultado, sin embargo, la configuración ocho (8), tiene un porcentaje de cobertura de $16.6 \%$, que es muy cercano a la configuración 6 , por lo que también se puede considerar relevante. La configuración 1, sugiere que para lograr patentes se requiere de "productividad" en las IES y de un contexto que incluya "innovación por unidad territorial", lo anterior, en ausencia de las variables: estructural, relacional, capital humano e investigación y acreditación de alta calidad, y, la ambigüedad de la 
variable unidad territorial de domicilio de las IES. La configuración 6 indica que se requiere "productividad" y "capital humano e investigación”, con ausencia de las demás variables y la configuración 8 , indica que se requiere del factor estructural e innovación por unidad territorial, en ausencia de las demás variables.

\section{Discusión}

La primera configuración, presenta la mayor cobertura por fila, lo que significa que el $28 \%$ de las IES de Colombia se identifica con esta solución. En esta configuración se destaca la importancia de la productividad, pues si las IES son intensivas en la producción de resultados de Investigación y Desarrollo (I+D) que a su vez condicionen a otros resultados, las IES cuentan con el factor de productividad (Berbegal-Mirabent \& Solé-Parellada, 2011; Olaya-Escobar et al., 2014), y estarán realizando KTT con gran impacto en la economía y la sociedad. La configuración 1 del modelo QCA, sugiere entonces, que para lograr patentes concedidas, las IES primero deben ser intensivas en resultados de investigación que impliquen la generación de otros, adicionalmente las IES deben encontrarse en un entorno o ambiente innovador, es decir que cuente con características de contexto socioeconómico que favorezca la innovación, estas características incluyen aspectos como, que las IES se encuentren conectadas con las tendencias de la tecnología (Siegel et al., 2003), con otras universidades de la región (Wright et al., 2008), con un entorno de desarrollo de ciencia e ingeniería y con cultura de apoyo comercial (O'Shea et al., 2005). Como ejemplo, en un caso hipotético de la realidad, si una IES busca obtener patentes en Colombia, primero genera resultados de investigación que condicionen a otros, ejemplo, las publicaciones científicas, las spin-off creadas, modelos de utilidad concedidos (Arenas \& González, 2018; Berbegal-Mirabent \& Solé-Parellada, 2011; Franco \& Haase, 2015; Olaya-Escobar et al., 2014) y lo más relevante la IES es intensiva en la solicitud de patentamiento, es decir, que entre más solicitudes de patentamiento realice, más posibilidades existen de que se le concedan patentes. Y segundo, se rodea de un entorno de innovación, pues para la IES es más favorable un entorno cercano que cuente con altos niveles de innovación dado que el desarrollo de ese contexto es mejor que en otras zonas donde los niveles de innovación son más bajos.

La segunda configuración relevante es la numero seis, e indica que si las IES son intensivas en productividad o resultados de $\mathrm{I}+\mathrm{D}$ que condicionan a otros resultados de investigación y a la vez son intensivas en capital humano e investigación, que se refiere al talento humano de las IES y a las aptitudes del personal (Berbegal-Mirabent \& Solé-Parellada, 2011; Olaya-Escobar et al., 2014) e incluye aspectos como investigadores académicos motivados, personal con experiencia en transferencia de tecnología (Chang et al., 2009), recompensas por la participación del profesorado (Siegel et al., 2003), administradores universitarios con características emprendedoras (Thursby \& Thursby, 2002), incentivos (Chapple et al., 2005), masa crítica en el equipo investigador (Wright et al., 2008), receptividad a contratos industriales (Thursby \& Thursby, 2002), proactividad, toma de riesgos, disponibilidad de tiempo (Chang et al., 2009), personal capacitado (Lockett \& Wright, 2005), calidad de los profesores (O'Shea et al., 2005), eminencia intelectual (Di Gregorio \& Shane, 2003) y perfil del investigador (Olaya-Escobar et al., 2020); las IES pueden lograr patentes concedidas. Esta configuración para las IES colombianas se presenta aunque las IES sean menos intensivas en el factor estructural, relacional, no tengan domicilio en Bogotá, no tengan acreditación de alta calidad y se encuentren en un entorno de poca innovación, así mismo, resalta la importancia de un capital humano representado principalmente por docentes e investigadores, que sea fuerte en aspectos como la formación del recurso humano, la generación de conocimiento y la apropiación social de conocimiento. Como ejemplo, en un caso hipotético, una IES de Colombia quiere lograr patentes concedidas, pero, aunque cuenta con altos niveles de productividad no se encuentra en un entorno o contexto de innovación como en el escenario de la Configuración 1, sin embargo, cuenta con un capital humano e investigación fuerte, que le permite apalancar el proceso de KTT para lograr patentes. En este caso la IES interesada en esta estrategia, una vez conocidas sus fortalezas y debilidades propias en cuanto a las variables descritas decide, encaminarse o no a fortalecer su productividad y su capital humano en alineación con su visión, misión, políticas, filosofía para lograr patentes.

La tercera configuración es la ocho, e indica que para que las IES logren patentes de invención concedidas, se requiere contar con una parte estructural fuerte y un entorno innovador para las IES, lo estructural se refiere a las características propias del funcionamiento de las IES (Berbegal-Mirabent \& Solé-Parellada, 2011; Olaya-Escobar et al., 2014), e incluye aspectos como, tener escuelas de ingeniería (Chang et al., 2009), escuelas de ciencias biológicas (Thursby \& Kemp, 2002), escuela de medicina y calidad de las facultades (Thursby et al., 2001), presencia de incubadoras (Chang et al., 2009), presencia de oficinas de transferencia (Markman et al., 2005), tener en cuenta el campo principal científico, posición en ranking de universidades, edad de las oficinas de transferencia, número de estudiantes y soporte organizacional en propiedad intelectual (Berbegal-Mirabent \& Solé-Parellada, 2011). En esta configuración se puede observar para el caso de las IES de Colombia que no es necesario que las IES tengan domicilio en Bogotá, dado que cuentan con una parte estructural robusta que les permite lograr la salida "patentes concedidas" desde otro domicilio pero que contenga un entorno innovador. Es relevante mencionar que Bogotá como capital de Colombia se consideró importante para el estudio porque tiene los actores del ecosistema de innovación más robustos del país, cuenta con las universidades más importantes e intensivas en $\mathrm{I}+\mathrm{D}$, tiene el mayor porcentaje de concentración de empresas grandes del país y las entidades de gobierno, además ha desarrollado varias iniciativas para generar y fortalecer el ecosistema de innovación porque cuenta con los intermediarios como Connect.

Por otro lado, al realizar la lectura vertical de los datos del modelo QCA presentado en la Tabla 6, se encuentra que el factor "relacional", asociado a las redes de contacto entre actores (Olaya-Escobar et al., 2014), sin estar catalogado como condición necesaria para generar patentes, está presente en seis de las diez configuraciones, lo que da a entender de forma general que es importante la relación entre actores Universidad-Empresa-Estado que se plantea en la literatura (Etzkowitz \& Leydesdorff, 2000; Johnson, 2008), para apoyar la 
generación de patentes concedidas, y a la vez la KTT desde las IES y la Innovación. Así mismo, con esta información, se destaca la importancia de la cooperación entre universidades (Chang \& Yang, 2008), y de la investigación cooperativa especialmente con empresas privadas (HAN \& KIM, 2016). También, según el contexto colombiano se encuentra relevante observar la composición de este factor, que indica que las coautorías en SCOPUS y las citaciones de artículos publicados en WOS y SCOPUS son recomendables para aportar de forma sustancial a la salida patentes de invención concedidas. Al continuar con la lectura vertical se evidencia que los factores estructural, productividad, capital humano e investigación, y la variable innovación por unidad territorial de las IES, están presentes cada una en cinco de las diez configuraciones, la variable acreditación de alta calidad en tres configuraciones y la de menor participación es la variable correspondiente a unidad territorial de domicilio de las IES con dos participaciones, la última variable mencionada presenta menor relevancia en participación, lo cual podría explicarse en el sentido que geográficamente existe un contexto de apertura y globalización.

\section{Conclusiones}

En la actualidad, la tercera misión de las universidades ha cobrado gran significado, pues con la KTT, las universidades se convierten en fuente de innovación capaz de realizar aportes valiosos a su entorno. En este sentido, las patentes de invención que se conceden a las IES tienen un valor económico relevante en el capital intelectual de las IES, son consideradas de alto impacto en las mediciones de innovación de los rankings que evalúan las universidades más innovadoras del mundo, son consideradas en la literatura como más globales que las patentes concedidas a las empresas porque generalmente son más citadas e implican otros resultados de investigación para su generación. Por lo anterior, y teniendo en cuenta que la tendencia actual de las universidades que son intensivas en generación de patentes (según la literatura) se orienta a la comercialización de este resultado de investigación, es importante para las IES aprovechar esta oportunidad y utilizar la opción de comercialización de las patentes como una de las fuentes de financiación.

Un aspecto relevante de este estudio es que las variables antecedentes propuestas en el modelo QCA, sin ser condiciones necesarias para generar "patentes de invención", al ser tratadas matemáticamente dentro del modelo propuesto, ofrecen un panorama de estrategias generales que pueden orientar la planeación de las IES que tengan como propósito fortalecer el patentamiento en Colombia. Respecto al análisis comparativo cualitativo (QCA), se construyó un modelo que presenta una relación de diez configuraciones que sirven como mecanismo para permitirle a las IES seleccionar estrategias de transferencia para obtener "patentes de invención". Dentro de la solución general se destaca la relevancia de la configuración número uno, que con una cobertura por fila de $28 \%$ relaciona ante las demás variables de entrada "la productividad" y "la innovación por unidad territorial" como mejor predictor del outcome. Así mismo, se evidencia que el factor con mayor presencia en las diez soluciones es el "relacional", lo que sugiere que en el contexto de las IES de Colombia la relación Universidad-Empresa-Estado es relevante. Se destaca que todas las configuraciones incluyen más de dos factores y que la cobertura y consistencia se encuentran sobre los umbrales establecidos en la literatura.

Se concluye que dependiendo de las características de cada Institución de Educación Superior de Colombia se pueden generar patentes de invención, tomando como referencia las diferentes rutas que se presentan y se sugieren en este modelo. Lo anterior abre las posibilidades para que cada institución plantee sus propias estrategias de acuerdo a sus fortalezas y debilidades en cuanto a las condiciones antecedentes identificadas en el modelo propuesto, esto, dado que no existe una única receta estandarizada para obtener patentes.

Por otro lado, respecto a trabajos futuros, se sugiere desarrollar revisiones de bases de datos adicionales para obtener información más robusta, del mismo modo realizar el estudio con fuentes de información primaria que permitan migrar del estudio documental al estudio de campo. Uno de los aspectos a tener presente con la recolección de información de fuente primaria puede ser el uso de amplios recursos económicos, que en la mayoría de los casos se puede convertir en una gran limitación. En cuanto al desarrollo del modelo con QCA, se podrían tomar otros resultados de investigación como outcome (licencia o acuerdo de regalías, investigación en colaboración, investigación por contrato y spin off-startups), en este caso una salida que está acorde con las tendencias de comercialización y emprendimiento universitario y que es fácilmente cuantificable y equiparable entre IES corresponde al número de spin-off, sin embargo, en Colombia apenas se empieza a dinamizar la generación de este output, razón por la cual aún no se encuentra muy representativo el número de spin-off generadas frente al número de IES existentes en el país.

De acuerdo al tema de investigación y la información obtenida con el desarrollo de este trabajo, se sugieren a continuación cuatro líneas futuras de investigación.

1. Identificación de factores determinantes para realizar emprendimiento académico desde las Instituciones de Educación Superior.

2. Identificación de factores determinantes para la comercialización de resultados de investigación desde las Instituciones de Educación Superior.

3. Diseño de modelos de emprendimiento académico que se ajusten a las características contextuales de las Instituciones de Educación Superior.

4. Diseño de modelos de comercialización de resultados de investigación que se ajusten a las características contextuales de las Instituciones de Educación Superior.

\section{Referencias}

Arenas, J., \& González, D. (2018). Technology Transfer Models and Elements in the University-Industry Collaboration. Administrative Sciences, 8(2), 19. https://doi.org/10.3390/admsci8020019 
Berbegal-Mirabent, J., \& Guerrero, A. D. (2016). Examining technology transfer activities at universities: Does one recipe explain all outcomes? 137-144.

Berbegal-Mirabent, J., \& Solé-Parellada, F. (2011). Caracterización del proceso de valorización de la I $+\mathrm{D}$ universitaria. 5th International Conference on Industrial Engineering and Industrial Management, $558-567$.

Bierly, P., \& Chakrabarti, A. (1996). Generic knowledge strategies in the U.S. pharmaceutical industry. Strategic Management Journal, 17(S2), 123-135. https://doi.org/10.1002/smj.4250171111

Bolisani, E., \& Bratianu, C. (2017). Knowledge strategy planning: an integrated approach to manage uncertainty, turbulence, and dynamics. Journal of Knowledge Management, 21(2), 233-253. https://doi. org/10.1108/JKM-02-2016-0071

Bradley, S. R., Hayter, C. S., \& Link, A. N. (2013). Models and Methods of University Technology Transfer. Foundations and Trends ${ }^{\circledR}$ in Entrepreneurship, 9(6), 571-650. https://doi.org/10.1561/0300000048

Brătianu, C. (2015). Developing Strategic Thinking in Business Education. Management Dynamics in the Knowledge Economy, 3(3), 409429. www.managementdynamics.ro

Budyldina, N. (2018). Entrepreneurial universities and regional contribution. International Entrepreneurship and Management Journal, 14(2), 265-277. https://doi.org/10.1007/s11365-018-0500-0

Carree, M., Piergiovanni, R., Santarelli, E., \& Verheul, I. (2015). Factors favoring innovation from a regional perspective: A comparison of patents and trademarks. International Entrepreneurship and Management Journal, 11(4), 793-810. https://doi.org/10.1007/s11365-014-0313-8

CEPAL. (2016). Ciencia, tecnología e innovación en la economía digital. La situación de América Latina y el Caribe. Segunda Reunión de La Conferencia de Ciencia, Innovación y TIC de La CEPAL, 96. https:// doi.org/10.1017/CBO9781107415324.004

Chang, Y.-C., Chen, M.-H., Hua, M., \& Yang, P. Y. (2006). Managing academic innovation in Taiwan: Towards a 'scientific-economic' framework. Technological Forecasting and Social Change, 73(2), 199-213. https://doi.org/10.1016/J.TECHFORE.2004.10.004

Chang, Y.-C., \& Yang, P. Y. (2008). The impacts of academic patenting and licensing on knowledge production and diffChang, Y.-C., \&

Yang, P. Y. (2008). The impacts of academic patenting and licensing on knowledge production and diffusion: a test of the anti-commons effect in Taiwan. R\&D Man. R\&D Management, 38(3), 321-334. https://doi.org/10.1111/j.1467-9310.2008.00513.x-i1

Chang, Y.-C., Yang, P. Y., \& Chen, M.-H. (2009). The determinants of academic research commercial performance: Towards an organizational ambidexterity perspective. Research Policy, 38(6), 936-946. https://doi.org/10.1016/J.RESPOL.2009.03.005
Chang, Y.-C., Yang, P. Y., Martin, B. R., Chi, H.-R., \& Tsai-Lin, T.-F. (2016). Entrepreneurial universities and research ambidexterity: A multilevel analysis. Technovation, 54, 7-21. https://doi.org/10.1016/J. TECHNOVATION.2016.02.006

Chapple, W., Lockett, A., Siegel, D., \& Wright, M. (2005). Assessing the relative performance of U.K. university technology transfer offices: parametric and non-parametric evidence. Research Policy, 34(3), 369-384. https://doi.org/10.1016/J.RESPOL.2005.01.007

Cornell University, INSEAD, \& WIPO. (2017). The Global Innovation Index 2017 Innovation Feeding the World. In The Global Innovation Index 2017: Innovation Feeding the World.

CRES. (2018a). El Papel Estratégico De La Educación Superior En El Desarrollo Sostenible De América Latina Y El Caribe. http://www. cres2018.org/uploads/educacion-superior-desarrollo-sostenible.pdf CRES. (2018b). Tendencias de la educación superior en América Latina y el Caribe 2018. http://www.cres2018.org/uploads/educacionsuperior-tendencias-ecuador.pdf

Di Gregorio, D., \& Shane, S. (2003). Why do some universities generate more start-ups than others? Research Policy, 32(2), 209-227. https://doi.org/10.1016/S0048-7333(02)00097-5

Eisenhardt, K. M., \& Santos, F. M. (2012). Knowledge-Based View: A New Theory of Strategy? In Handbook of Strategy and Management (pp. 139-164). SAGE Publications Ltd. https://doi. org/10.4135/9781848608313.n7

Etzkowitz, H. (2003). Innovation in Innovation: The Triple Helix of University-Industry-Government Relations. Social Science Information, 42(3), 293-337. https://doi.org/10.1177/05390184030423002

Etzkowitz, H., \& Leydesdorff, L. (2000). The dynamics of innovation: from National Systems and "Mode 2" to a Triple Helix of university-industry-government relations. Research Policy, 29(2), 109-123. https://doi.org/10.1016/S0048-7333(99)00055-4

Etzkowitz, H., Webster, A., Gebhardt, C., \& Terra, B. R. C. (2000). The future of the university and the university of the future: evolution of ivory tower to entrepreneurial paradigm. Research Policy, 29(2), 313-330. https://doi.org/10.1016/S0048-7333(99)00069-4

Fernández Guerrero, R., Revuelto Taboada, L., \& Simón Moya, V. (2018). Supervivencia de empresas sociales de nueva creación. Un enfoque basado en el análisis cualitativo comparativo fsQCA. CIRIECEspaña, Revista de Economía Pública, Social y Cooperativa. https:// doi.org/10.7203/CIRIEC-E.92.10735

Figueroa-Galvis, N. Y., Olaya-Escobar, E.S., \& Castro Silva, H. (2019a). Identificación y comparación de factores que inciden en la transferencia de tecnología - XVIII Congreso Latino Iberoamericano de Gestión Tecnológica ALTEC 2019 Medellín. Debates Sobre Innovación. http://economiaeinnovacionuamx.org/secciones/debatessobre$\% 0$ Ainnovacion 
Figueroa-Galvis, N. Y., Olaya-Escobar, E. S., \& Castro Silva, H. (2019b). Diseño de un modelo para la selección de estrategias de transferencia a la medida de las características contextuales de las Instituciones de Educación Superior: caso Colombia. Universidad Pedagógica y Tecnológica de Colombia.

Fiss, P. C. (2011). Building Better Causal Theories: A Fuzzy Set Approach to Typologies in Organization Research. Academy of Management Journal, 54(2), 393-420. https://doi.org/10.5465/ amj.2011.60263120

Franco, M., \& Haase, H. (2015). University-industry cooperation: Researchers' motivations and interaction channels. Journal of Engineering and Technology Management - JET-M, 36, 41-51. https://doi. org/10.1016/j.jengtecman.2015.05.002

Fuquen, H. S., \& Olaya-Escobar, E. S. (2018). A technology transfer strategy based on the dynamics of the generation of intellectual property in Latin-America. Intangible Capital, 14(2), 203-252. https:// doi.org/10.3926/ic.873

Gandini, L. (2014). El análisis comparativo cualitativo como estrategia metodológica. June.

Hamdoun, M., Chiappetta Jabbour, C. J., \& Ben Othman, H. (2018). Knowledge transfer and organizational innovation: Impacts of quality and environmental management. Journal of Cleaner Production, 193, 759-770. https://doi.org/10.1016/J.JCLEPRO.2018.05.031

Han, J. (2017). Technology commercialization through sustainable knowledge sharing from university-industry collaborations, with a focus on patent propensity. Sustainability (Switzerland), 9(10). https:// doi.org/10.3390/su9101808

HAN, J., \& KIM, J. (2016). EMPIRICAL ANALYSIS OF TECHNOLOGY TRANSFER IN KOREAN UNIVERSITIES. International Journal of Innovation Management, 20(08), 1640018. https://doi. org/10.1142/S1363919616400181

Hogan, T. (2011). an Overview of the Knowledge Economy, With a Focus on. August, 1-35.

Johnson, W. H. A. (2008). Roles, resources and benefits of intermediate organizations supporting triple helix collaborative R\&amp;D: The case of Precarn. Technovation, 28(8), 495-505. https://doi. org/10.1016/J.TECHNOVATION.2008.02.007

Kashyap, A., \& Agrawal, R. (2019). Scale development and modeling of intellectual property creation capability in higher education. Journal of Intellectual Capital, 21(1), 115-138. https://doi.org/10.1108/ JIC-09-2018-0168

Kasten, J. (2007). Knowledge strategy and its influence on knowledge organization (Vol. 1). http://dlist.sir.arizona.edu/1907
Landry, R., Amara, N., Cloutier, J. S., \& Halilem, N. (2013). Technology transfer organizations: Services and business models. Technovation, 33(12), 431-449. https://doi.org/10.1016/j.technovation.2013.09.008

Laredo, P. (2007). Revisiting the Third Mission of Universities: Toward a Renewed Categorization of University Activities? Higher Education Policy, 20(4), 441-456. https://doi.org/10.1057/palgrave.hep.8300169

Linton, J. D. (2018). DNA of the Triple Helix: Introduction to the special issue. Technovation. https://doi.org/10.1016/J.TECHNOVATION.2018.07.002

Lockett, A., \& Wright, M. (2005). Resources, capabilities, risk capital and the creation of university spin-out companies. Research Policy, 34(7), 1043-1057. https://doi.org/10.1016/J.RESPOL.2005.05.006

Lockett, A., Wright, M., \& Wild, A. (2015). The Institutionalization of Third Stream Activities in UK Higher Education: The Role of Discourse and Metrics. British Journal of Management, 26(1), 78-92. https://doi.org/10.1111/1467-8551.12069

Longest, K. C., \& Vaisey, S. (2008). fuzzy: A program for performing qualitative comparative analyses (QCA) in Stata. Stata Journal, 8(1), 79-104. https://ideas.repec.org/a/tsj/stataj/v8y2008i1p79-104.html

Markman, G. D., Phan, P. H., Balkin, D. B., \& Gianiodis, P. T. (2005). Entrepreneurship and university-based technology transfer. Journal of Business Venturing, 20(2), 241-263. https://doi.org/10.1016/J. JBUSVENT.2003.12.003

Mintzberg, H. (1993). The Fall and Rise of Strategic Planning. Harvard Business Review.

O'Shea, R. P., Allen, T. J., Chevalier, A., \& Roche, F. (2005). Entrepreneurial orientation, technology transfer and spinoff performance of U.S. universities. Research Policy, 34(7), 994-1009. https://doi. org/10.1016/j.respol.2005.05.011

Olaya-Escobar, E. S., Berbegal-Mirabent, J., \& Alegre, I. (2020). Technological Forecasting \& Social Change Exploring the relationship between service quality of technology transfer offices and researchers ' patenting activity. Technological Forecasting \& Social Change, 157(November 2018), 120097. https://doi.org/10.1016/j.techfore.2020.120097

Olaya-Escobar, E. S., Berbegal-Mirabent, J., \& Duarte, O. G. (2014). Desempeño de las oficinas de transferencia universitarias como intermediarias para la potencialización del mercado de conocimiento. Intangible Capital, 10(1), 155-188. https://doi.org/http://dx.doi. org/10.3926/ic.497

OMPI. (2019). World Intellectual Property Indicators 2019.

Philpott, K., Dooley, L., O’Reilly, C., \& Lupton, G. (2011). The entrepreneurial university: Examining the underlying academic tensions. Technovation, 31(4), 161-170. https://doi.org/10.1016/J.TECHNOVATION.2010.12.003 
Pinheiro, R., Langa, P. V., \& Pausits, A. (2015). The institutionalization of universities' third mission: introduction to the special issue. European Journal of Higher Education, 5(3), 227-232. https://doi.org/ $10.1080 / 21568235.2015 .1044551$

Ragin, C. (2006). Guía del usuario de Fuzzy-Set / Qualitative Comparative Analysis 2.0. Tucson, Arizona: Departamento de Sociología, Universidad de Arizona.

Ragin, C. (2008). Redesigning social inquiry: fuzzy sets and beyond. University of Chicago Press.

Ragin, C., Drass, K. A., \& Sean, D. (2006). Fuzzy-Set / Qualitative Comparative Analysis 2.0 . Tucson, Arizona: Departamento de Sociología, Universidad de Arizona.

Ragin, C., \& Fiss, P. C. (2008). Net effects analysis versus configurational analysis: An empirical demonstration. In Redesigning Social Inquiry: Set Relations in Social Research. In press. Chicago, University of Chicago Press.

Reuters. (2018). Reuters Top 100: las universidades más innovadoras del mundo - 2018 - Reuters. https://www.reuters.com/article/usamers-reuters-ranking-innovative-univ/reuters-top-100-the-worldsmost-innovative-universities-2018-idUSKCN1ML0AZ\#unis

Schmid, J., \& Fajebe, A. (2019). Variation in patent impact by organization type: An investigation of government, university, and corporate patents. Science and Public Policy, 46(4), 589-598. https://doi. org/10.1093/scipol/scz010

Schneider, C. Q., \& Wagemann, C. (2010). Standards of Good Practice in Qualitative Comparative Analysis (QCA) and Fuzzy-Sets. Comparative Sociology, 9(3), 397-418. https://doi.org/10.1163/15691321 0X12493538729793

Shattock, M., Unesco., \& International Institute for Educational Planning. (2009). Entrepreneurialism in universities and the knowledge economy : diversification and organizational change in European higher education. Society for Research into Higher Education \& Open University Press.

Siegel, D. S., Waldman, D., \& Link, A. (2003). Assessing the impact of organizational practices on the relative productivity of university technology transfer offices: an exploratory study. Research Policy, 32(1), 27-48. https://doi.org/10.1016/S0048-7333(01)00196-2
The World Bank. (2018). How does the World Bank classify countries? - World Bank Data Help Desk. https://datahelpdesk.worldbank.org/ knowledgebase/articles/378834-how-does-the-world-bank-classifycountries

Thursby, J. G., Jensen, R., \& Thursby, M. C. (2001). Objectives, Characteristics and Outcomes of University Licensing: A Survey of Major U.S. Universities. The Journal of Technology Transfer, 26(1/2), 59-72. https://doi.org/10.1023/A:1007884111883

Thursby, J. G., \& Kemp, S. (2002). Growth and productive efficiency of university intellectual property licensing. Research Policy, 31(1), 109-124. https://doi.org/10.1016/S0048-7333(00)00160-8

Thursby, J. G., \& Thursby, M. C. (2002). Who Is Selling the Ivory Tower? Sources of Growth in University Licensing. Management Science, 48(1), 90-104. https://doi.org/10.1287/mnsc.48.1.90.14271

Toscano, F. L. P., Mainardes, E. W., \& Lasso, S. V. (2017). Exploring Challenges in University Technology Transfer in Brazil. International Journal of Innovation and Technology Management, 14(04), 1750021. https://doi.org/10.1142/S0219877017500213

Von Krogh, G., Nonaka, I., \& Aben, M. (2001). Making the most of your company's knowledge: A strategic framework. Long Range Planning, 34(4), 421-439. https://doi.org/10.1016/S0024-6301(01)00059-0

WIPO. (2020). Patentes académicas: cómo las universidades y las organizaciones públicas de investigación están utilizando su propiedad intelectual para impulsar la investigación y estimular nuevas empresas innovadoras. https://www.wipo.int/sme/en/documents/academic_ patenting.html

Woodside, A. G. (2012). Proposing a new logic for data analysis in marketing and consumer behavior: case study research of large- $\mathrm{N}$ survey data for estimating algorithms that accurately profile X (extremely high-use) consumers. Journal of Global Scholars of Marketing Science, 22(4), 277-289. https://doi.org/10.1080/21639159.2012.717369

Wright, M., Clarysse, B., Lockett, A., \& Knockaert, M. (2008). Midrange universities' linkages with industry: Knowledge types and the role of intermediaries. Research Policy, 37(8), 1205-1223. https://doi. org/10.1016/J.RESPOL.2008.04.021

Zack, M. H. (1999). Developing a Knowledge Strategy. In CALIFORNIA MANAGEMENT REVIEW (Vol. 41, Issue 3). 
